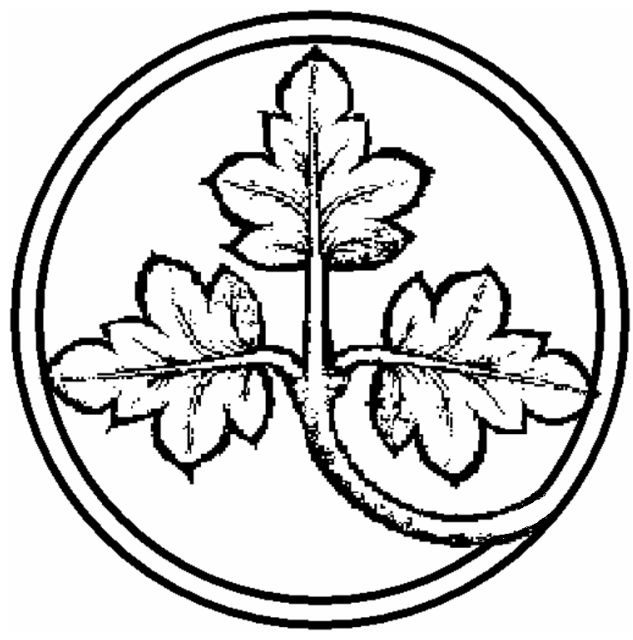

\author{
Preprints of the \\ Max Planck Institute \\ for Research on Collective Goods \\ Bonn \\ 2005/8
}

Altruism and charitable giving in a fully replicated economy

Thomas Gaube 


\section{Altruism and charitable giving in a fully replicated economy}

Thomas Gaube

April 2005 


\title{
Altruism and charitable giving in a fully replicated economy
}

\author{
Thomas Gaube*
}

This version: October 2005

\begin{abstract}
In this paper, an economy is analyzed where one group of agents, the altruists, cares about the well-being of another group of agents, the recipients. It is asked how changes in the size of these groups affect the altruists' charitable giving in the Nash equilibrium. I show that a pure group size effect, i.e., a proportional expansion of both subgroups can lead to less free riding and to a lower degree of underprovision relative to the efficient level of charitable giving.
\end{abstract}

Keywords: altruism, public goods, group size, charitable giving

JEL-Classification: D64, H41

To appear:

Journal of Public Economics

*Max Planck Institute for Research on Collective Goods, Kurt-Schumacher-Str. 10, D-53113 Bonn, Germany, Tel.: +49-228-9141670, fax: +49-228-9141655, E-mail adress: gaube@mpprdg.mpg.de 


\section{Introduction}

In this paper, an economy is analyzed where one group of agents, the altruists, cares about the well-being of another group of agents, the recipients. It is asked how changes in the size of these groups affect the altruists' charitable giving in the Nash equilibrium. Particular attention is given to the question whether replications of the whole economy increase or decrease (i) per-capita donations, (ii) the ratio between contributors and free riders, and (iii) the ratio between the equilibrium level and the efficient level of charitable giving. This question is motivated by a closely related topic of the literature, namely the effect of an increase in group size on private donations to a public good. In this context, it has been shown by means of the voluntary contribution model of public good provision that the variables (i), (ii), and (iii) are negatively correlated with group size, under mild assumptions concerning the individuals' preferences. ${ }^{1}$ These findings on public good provision thus confirm Olson's (1965) claim that the free-rider problem of voluntary donations becomes more severe as group size increases.

The voluntary contribution model of public good provision has been interpreted in two different ways. First, in the literal sense, namely that a nonrival commodity like public broadcasting is provided. In terms of this interpretation, the model relies on the implicit assumption that the individuals contribute to the public good only because of their personal interest in consuming this commodity. Hence, altruistic motives play no role. Still, following Becker (1974), the same model is employed also for investigating altruistically motivated donations. The proposed analogy between altruistic giving and (selfish) public good provision is based on the idea that an altruist's donation to another individual is equivalent to a public good because the other altruists become better off as well. Therefore, the public good model has been used for analyzing any form of private donations which can broadly be classified as a contribution to 'good causes'. From this perspective, the group-size results of the literature thus suggest that pub-

\footnotetext{
${ }^{1}$ Generalizing earlier results by McGuire (1974) and Chamberlin (1974), Andreoni (1988), and Fries et al. (1991) have shown that the variables (i) and (ii) are negatively correlated with group size if private consumption and the public good are normal commodities. Gaube (2001) has pointed out that the additional assumption of gross substitutability between these commodities is sufficient for a negative effect of group size on the variable (iii).
} 
lic intervention into the market equilibrium for charitable giving becomes more desirable as group size is increased.

The present paper argues that the comparison between voluntary donations in a small and a large community may be different for the case of altruistically motivated giving (i.e., charitable giving in the literal sense) than for the case of selfish donations to a public good. The reasoning is as follows: Consider a community where a local radio station is financed by voluntary donations. If group size in this community is increased by introducing replicas of each agent, the agents' utility from consuming the public good does not change, and all contributors reduce their donations in the Nash equilibrium because they correctly anticipate that their replicas will contribute to the public good as well. In contrast, assume now that a group of altruists supports a group of individuals who are in need of an expensive medical treatment. Since charitable giving is nonrival from the altruists' perspective, the two examples are formally equivalent as long as a change in group size is modelled by replicating only the group of altruists. However, if the whole economy is replicated, charitable giving has to be allocated among a larger number of individuals because the monetary transfers are perfectly rival from the recipients' perspective. Hence, as long as the altruists care about single individuals and not just about the aggregate donation, they will take the increased number of recipients into account. Therefore, an increase in group size may affect charitable giving differently than a model where the aggregate donation is nonrival for all agents in the economy suggests.

The subsequent analysis aims to explore the consequences of this argument in more detail. For that purpose, altruistic preferences are not expressed indirectly by introducing a public good but directly by assuming that an altruist's utility depends on the well-being of each recipient. This framework allows us to investigate how changes in the number of altruists and recipients affect charitable giving in the Nash equilibrium. It is shown that the altruists' per-capita donation and the ratio between contributors and non-contributors can increase in group size provided that replications of the whole economy are considered. For the case of additively separable altruistic preferences, a neutrality result is established according to which the equilibrium allocation in per-capita terms does not change if the number of altruists and recipients is increased proportionally. Similar find- 
ings are obtained with respect to the ratio between the equilibrium level and the efficient level of charitable giving: It is shown that this ratio can also increase in group size and may even converge to unity if the economy becomes sufficiently large.

These findings point out that altruistic charitable giving can react quite differently to a change in group size than selfish donations to a public good. As argued above, the difference in results stems from the fact that charitable giving is nonrival only from the altruists' but not from the recipients' perspective. In order to work out this point most clearly, I consider a framework where a strict separation is made between donors and recipients. The model thus differs from the setup of linear public good experiments, where monetary transfers among all participants take place such that givers and recipients are the same persons. Note, however, that altruism is likely to be relevant in such experiments as well: Anderson et al. (1998) and Goeree et al. (2002) have shown that altruism can explain why a non-positive correlation between group size and free riding has been observed in several public good experiments. ${ }^{2}$ They argue that a larger group size gives altruistic participants an incentive to increase their donations because the number of agents who benefit from these donations is increased. ${ }^{3}$ The subsequent analysis makes a similar point. However, I consider only an altruistic link between the donors and the recipients, not among the donors themselves. Therefore, the paper does not rely on the idea that the social return of a nonrival commodity increases with group size, but on the observation that charitable donations are nonrival only from the altruists' perspective. In addition, Anderson et al. (1998) and Goeree et al. (2002) focus on linear payoffs and additively separable utility functions, whereas the present study investigates a nonlinear model, where donations and private consumption are imperfect substitutes, and where no specific utility function is assumed from the outset. Accordingly, interior Nash equilibria

\footnotetext{
${ }^{2}$ Isaac and Walker (1988), Isaac et al. (1994), and Goeree et al. (2002) report public good experiments where group size and free riding are negatively correlated. The empirical findings of Lipford (1996), Brunner (1998), and Haan and Kooreman (2002), which deal with contributions to churches, broadcasting, and candy bars respectively, also raise doubts that the free-rider problem of voluntary public good provision becomes more severe as more agents enter the economy.

${ }^{3}$ This argument is also briefly explored in Andreoni and Miller (2002).
} 
and interior efficient allocations are analyzed, and it is shown that the specific form of altruistic preferences is crucial for answering the question whether group size and free riding are positively correlated. ${ }^{4}$

The remainder of this paper is organized as follows. Section 2 presents the model. In Section 3, the link between group size, per-capita donations, and free riding incentives is analyzed. Section 4 deals with the effect of group size on the ratio between the equilibrium level and the efficient level of charitable giving. Section 5 concludes.

\section{The model}

Consider an economy which consists of two groups of agents, namely $I \geq 1$ altruists and $K \geq 1$ recipients. The recipients $k=1, \ldots, K$ have no initial wealth and rely on monetary transfers $b_{k}$, which are financed by the altruists. It is assumed that the well-being of each recipient is monotonically increasing in $b_{k}$. An altruist of type $i \in\{1, \ldots, I\}$ has monetary endowment $\omega_{i}>0$, which can be spent for private consumption $x_{i}$ and charitable giving $g_{i}$. The sum $G$ of these donations is allocated among the recipients such that $G=\sum_{k=1}^{K} b_{k}$. It is assumed that the altruists' preferences can be represented by quasiconcave utility functions

$$
\tilde{U}^{i}\left(x_{i}, g_{i}, \tilde{a}^{K}\left(b_{1}, \ldots, b_{K}\right)\right),
$$

which are increasing in $x_{i}\left(\tilde{U}_{x}^{i}>0\right)$, non-decreasing in $g_{i}\left(\tilde{U}_{g}^{i} \geq 0\right)$, and increasing in $b_{k}\left(\tilde{U}_{k}^{i}>0\right), k=1, \ldots, K$, at least for transfers below some threshold $\bar{b}>0$. The last of these assumptions means that all types $i$ care about the well-being of all recipients $k$. Hence, each transfer $b_{k}$ is a public good from the altruists' perspective. However, the analysis does not rule out the possibility that altruism fades away if the transfers $b_{k}$ become sufficiently large, i.e., if they exceed some threshold $\bar{b}^{5}$ The assumption $\tilde{U}_{g}^{i} \geq 0$ takes into account that charitable giving

\footnotetext{
${ }^{4}$ While the present paper does not intend to explain empirical observations concerning the private provision of public goods, the subsequent findings make it clear that the functional form of the altruists' utility functions is crucial in this context as well.

${ }^{5}$ None of the propositions derived below hinge on the assumption that such a threshold exists. Some of the illustrative examples, however, makes use of the possibility that the altruists' utility is increasing in $b_{k}$ only for transfers below some finite threshold $\bar{b}$.
} 
may also provide private utility in the sense of Andreoni's (1990) warm-glow model of impure altruism. In terms of this model, pure altruists care only about charitable giving ( $b_{k}$ in the present context) and not about their own contributions $g_{i}$, whereas impure altruists care about both. Andreoni (1990) and Ribar and Wilhelm (2002) show that the impure-altruism model is better suited for explaining observed patterns of charitable giving than the basic voluntary contribution model, where no private motive $g_{i}$ is taken into account. Note, however, that I do not rule out the case $\tilde{U}_{g}^{i}=0$. The subsequent results thus hold in the absence of a warm-glow effect as well.

In terms of the utility functions (1), a change in the number of recipients $K$ can be interpreted as a change in the number of public goods. In order to obtain a framework which is as close as possible to the standard model of a single privately supplied public good, I will assume that the derivatives $\tilde{a}_{k}^{K}(\cdot)$ of the functions $\tilde{a}^{K}(\cdot)$ satisfy the conditions

$$
\left.\frac{\tilde{a}_{k}^{K}(\cdot)}{\tilde{a}_{l}^{K}(\cdot)}\right|_{b_{k}=b_{l}}=1,\left.\quad \frac{\tilde{a}_{k}^{K}(\cdot)}{\tilde{a}_{l}^{K}(\cdot)}\right|_{b_{k}>b_{l}} \leq 1, \quad \forall k, l \in\{1, \ldots, K\} .
$$

These conditions mean that the altruists are either indifferent with respect to the distribution of the aggregate transfer $G=\sum_{k=1}^{K} b_{k}$ among the recipients, or that they prefer an allocation where each recipient obtains the average donation $b:=G / K$. This can best be interpreted as "anonymity of recipients": The donors do not like one specific recipient more than others. Assumption (2) implies that we can restrict attention to those allocations where transfers are symmetric, such that only the average transfer $b$ and the length $K$ of the vector $\left(b_{1}, \ldots, b_{K}\right)$ are relevant for the donors. In order to formalize this property, consider the functions $a^{K}(b, K):=\left.\tilde{a}^{K}\left(b_{1}, \ldots, b_{K}\right)\right|_{b_{k}=b \forall k}$. Because of $b=G / K$, these functions can be used to express the donors' preferences by means of

$$
U^{i}\left(x_{i}, g_{i}, G, K\right):=\tilde{U}^{i}\left(x_{i}, g_{i}, a^{K}(G / K, K)\right) .
$$

In the following, a change in group size is analyzed by considering replications of an initial economy that consists of the $I \geq 1$ altruists and in which the number of recipients $K$ is normalized to unity. The subgroup of altruists and the subgroup of recipients is increased by introducing $N-1 \geq 0$ replicas of each altruist $i \in\{1, \ldots, I\}$ and $K-1 \geq 0$ replicas of the single recipient, respectively. 
Aggregate charitable giving in an economy with $N I$ altruists thus amounts to $G=N\left(\sum_{i=1}^{I} g_{i}\right)$. I will distinguish between partial replications of the economy, where either $N$ or $K$ is changed, and full replications of the economy, where $N$ and $K$ are increased proportionally such that the initial ratio $I$ between altruists and recipients remains unaffected.

When deciding upon $x_{i}$ and $g_{i}$, an altruist takes the aggregate provision $G_{-i}:=$ $G-g_{i}$ of all other altruists as given. ${ }^{6}$ Since $g_{i}$ and $x_{i}$ have to satisfy the budget constraint $\omega_{i}-x_{i}-g_{i}=0$, each altruist maximizes $U^{i}\left(\omega_{i}-g_{i}, g_{i}, G_{-i}+g_{i}, K\right)$ with respect to $g_{i}$. The first-order condition of those agents who choose a strictly positive amount $g_{i}$ in the Nash equilibrium can thus be written in the form $-U_{x}^{i}+U_{g}^{i}+U_{G}^{i} \geq 0$, where strict inequality implies $g_{i}=\omega_{i}$ and $x_{i}=0$. Conversely, we have $-U_{x}^{i}+U_{g}^{i}+U_{G}^{i} \leq 0$ for the free riders. In the following, I will assume that private consumption $x_{i}$ and the marginal utility $U_{G}^{i}$ are strictly positive for all types $i$ in equilibrium. ${ }^{7}$ These assumptions imply that $-U_{x}^{i}+U_{g}^{i}+U_{G}^{i}=0$ holds for the contributors and that $U_{x}^{i}>U_{g}^{i}$ holds for all individuals. The contributors' first-order conditions can thus be expressed in terms of the marginal rate of substitution

$$
m_{i}\left(x_{i}, g_{i}, G, K\right):=\frac{U_{G}^{i}}{U_{x}^{i}-U_{g}^{i}}=1 .
$$

Accordingly, one obtains $m_{i}\left(\omega_{i}, 0, G, K\right) \leq 1$ for the free riders. Maximization of utility $U^{i}\left(\omega_{i}-g_{i}, g_{i}, G_{-i}+g_{i}, K\right)$ with respect to $g_{i}$ leads to the individual's reaction function, which can be written in the form

$$
g_{i}=\operatorname{Max}\left\{0, f_{i}\left(\omega_{i}+G_{-i}, G_{-i}, K\right)-G_{-i}\right\}
$$

Note that (5) is equivalent to the familiar reaction function of the warm-glow model, except that it depends on the number of recipients $K$. As in most contributions on voluntary public good provision, the subsequent analysis relies on the assumption that $x_{i}$ and $G$ are normal goods, and that the 'demand' for a warm glow is non-inferior. As explained in Andreoni (1990), these assumptions mean (i)

\footnotetext{
${ }^{6}$ Note that $G_{-i}$ does not only encompass the donations $g_{j}$ of the types $j \neq i$, but also the donations $g_{i}$ of the other $(N-1)$ individuals of type $i$.

${ }^{7}$ The assumption $U_{G}^{i}>0$ means that the (impure) altruists are not pure egoists in the sense of Andreoni (1990); that is, agents who are interested only in 'private' consumption $x_{i}$ and $g_{i}$, but not in the well-being of the recipients.
} 
$f_{1}^{i}>0$, (ii) $f_{2}^{i} \geq 0$, and (iii) $f_{1}^{i}+f_{2}^{i}<1$, where $f_{1}^{i}$ and $f_{2}^{i}$ are the derivatives of $f^{i}(\cdot)$ with respect to the first and the second argument, respectively. The inequalities (i) - (iii) can be replaced by means of the assumption that the marginal rates of substitution $m_{i}(\cdot)>0$ are increasing in $x_{i}$, decreasing in $G$, and non-increasing in $g_{i} .{ }^{8}$ Hence,

$$
\frac{\partial m_{i}(\cdot)}{\partial x_{i}}>0, \quad \frac{\partial m_{i}(\cdot)}{\partial G}<0, \quad \frac{\partial m_{i}(\cdot)}{\partial g_{i}} \leq 0, \quad \forall i \in\{1, \ldots, I\} .
$$

The normality assumption (6) implies that a unique Nash equilibrium $\left(g_{1}^{v}(N, K), \ldots, g_{I}^{v}(N, K)\right)$ exists for any pair of the group-size parameters $N$ and $K{ }^{9}$ Let $C^{v}(N, K)$ denote the set of contributors, i.e., of those types $i$ who choose $g_{i}^{v}(N, K)>0$ in an economy with parameters $N, K$. The number of contributing types is denoted by $\# C^{v}(N, K) \leq I$. The aggregate voluntary contribution, the per-capita voluntary contribution, and proportion of contributors among the altruists can then be expressed by means of $G^{v}(N, K):=N\left(\sum_{i=1}^{I} g_{i}^{v}(N, K)\right)$, $g^{v}(N, K):=G^{v}(N, K) /(N I)$, and $c^{v}(N, K):=\# C^{v}(N, K) / I$ respectively.

Note that a change of the parameters $N, K$ can influence the individuals' contributions $g_{i}^{v}(N, K)$ only if the first-order conditions $m_{i}\left(x_{i}, g_{i}, G, K\right)=1$ for the contributors or $m_{i}\left(x_{i}, g_{i}, G, K\right) \leq 1$ for the non-contributors are affected. Hence, if these inequalities are strict for all types $i$, i.e., if no altruist chooses an interior donation $\left.g_{i} \in\right] 0, \omega_{i}\left[\right.$ in equilibrium, the vector $\left(g_{1}^{v}(N, K), \ldots, g_{I}^{v}(N, K)\right)$ may not react to small changes of $N$ and $K$ for trivial reasons. In order to avoid multiple repetitions of this technical qualification, I will assume

$$
\exists i \in\{1, \ldots, I\}: \quad 0<g_{i}^{v}(N, K)<\omega_{i}
$$

${ }^{8}$ It is straightforward to show that the inequalities (i)-(iii) follow from (6). It can also easily be established that (i)-(iii) lead to $\left(\partial m_{i} / \partial G\right)\left(\partial m_{i} / \partial x_{i}\right) \leq 0$ and $\left(\partial m_{i} / \partial g_{i}\right)\left(\partial m_{i} / \partial x_{i}\right) \leq 0$. These inequalities imply (6) because utility is strictly quasiconcave. Note that $U_{g}^{i}=0$ and $\partial m_{i} / \partial g_{i}=0$ must hold if no warm-glow effect takes place. In this case, the first two inequalities in (6) are equivalent to the familiar conditions $U_{G x}^{i} U_{x}^{i}-U_{x x}^{i} U_{G}^{i}>0$ and $U_{G G}^{i} U_{x}^{i}-U_{x G}^{i} U_{G}^{i}<0$, which stand for normality of $G$ and $x_{i}$ respectively (see, e.g., Buchholz and Peters (2001), p. $65)$.

${ }^{9}$ Consider the slope $\partial g_{i} / \partial G_{-i}$ of the reaction function (5). As noted above, assumption (6) leads to the inequalities (i) - (iii), which in turn imply $0>\partial g_{i} / \partial G_{-i}>-1$ for $g_{i}>0$, and $\partial g_{i} / \partial G_{-i}=0$ for $g_{i}=0$. Andreoni (1990) notes that these properties are sufficient for existence and uniqueness of the Nash equilibrium. A formal proof of this claim can be found in Cornes et al. (1999). 
from the outset. This assumption implies that $m_{i}\left(x_{i}, g_{i}, G, K\right)=1$ holds for at least one type $i \in C^{v}(N, K)$, but it does not require that a specific type $i \in\{1, \ldots, I\}$ is a contributor irrespective of $N$ and $K$.

In terms of positive analysis, the link between group size and free riding is usually discussed by means of the variables $g^{v}(\cdot)$ and $c^{v}(\cdot)$. From a normative perspective, however, the comparison between the equilibrium provision $G^{v}(N, K)$ and efficient charitable giving $G^{*}(N, K)$ is of interest as well. Therefore, an efficiency benchmark has to be determined. In the following, efficient allocations will be characterized by means of the Samuelson condition

$$
N \sum_{i=1}^{I} m_{i}\left(x_{i}, g_{i}, G, K\right)=1
$$

Two comments with respect to this condition are in order. First, note that each allocation which satisfies (8) may correspond to a different provision level $G^{*}(\cdot)$. Therefore, one of these allocations has to be chosen. Following the analysis in Gaube (2001), I will employ the Lindahl allocation as a reference point for efficient charitable giving. ${ }^{10}$ In the present context, the Lindahl equilibrium is defined as follows: The agents of type $i=1, \ldots, I$ maximize utility $U^{i}\left(x_{i}, g_{i}, G, K\right)$ subject to the budget constraint $\omega_{i}-x_{i}-p_{i} G \geq 0$, where $p_{i}$ is the personalized price of the public good. In addition, they take into account that their personal contribution to the public good equals $g_{i}=p_{i} G$. In this way, the demand functions $x_{i}\left(p_{i}, \omega_{i}, K\right)$ and $G_{i}\left(p_{i}, \omega_{i}, K\right)$ are obtained. In equilibrium, the conditions $G_{1}(\cdot)=G_{2}(\cdot)=$ $\ldots=G_{I}(\cdot)$ and $N \sum_{i=1}^{I} p_{i}=1$ have to be satisfied. Accordingly, the efficient provision level is defined by means of $G^{*}(N, K):=G_{i}\left(p_{i}^{*}(N, K), \omega_{i}, K\right)$, where $p_{i}^{*}(N, K)$ is the Lindahl price for the agents of type $i \in\{1, \ldots, I\}$.

It should be noted also that the Samuelson condition (8) takes only the utility of the altruists, but not of the recipients into account. Clearly, a Pareto improvement among the recipients can be obtained if and only if the aggregate transfer $G=\sum_{k=1}^{K} b_{k}$ is increased. Hence, if we consider an allocation where the inequality $N \sum_{i=1}^{I} m_{i}\left(x_{i}, g_{i}, G, K\right)>1$ holds, an increase in $G$ can be used to make all

\footnotetext{
${ }^{10}$ This reference point will be used for investigating the index of easy riding $G^{v}(\cdot) / G^{*}(\cdot)$ introduced by Cornes and Sandler $(1984,1996)$. Their analysis deals with identical agents and defines the ratio $G^{v}(\cdot) / G^{*}(\cdot)$ by means of the symmetric efficient allocation. With identical agents, the Lindahl allocation and the symmetric efficient allocation coincide.
} 
types $i=1, \ldots, I$ and all agents $k=1, \ldots, K$ better off. However, allocations with $N \sum_{i=1}^{I} m_{i}\left(x_{i}, g_{i}, G, K\right)<1$ can also be Pareto efficient from the perspective of the whole economy because a reduction in $G$ necessarily harms at least one of the recipients $k \in\{1, \ldots, K\}$. In the present context, the quantity $G^{*}(N, K)$ should thus be interpreted as the minimal amount of Pareto efficient charitable giving. ${ }^{11}$ Therefore, I use the term "efficient provision" and do not refer to the Pareto concept.

\section{$3 \quad$ Free riding and easy riding}

This section is devoted to the question of how changes in group size affect the proportion of contributors $c^{v}(N, K)$ and the per-capita contribution $g^{v}(N, K)$ in the Nash equilibrium. For the voluntary contribution model of public good provision, where it is assumed that the preferences of all agents can be expressed by means of utility functions $U^{i}\left(x_{i}, G\right)$, this topic has been analyzed, for example, in Fries et al. (1991). They show that the proportion of contributing agents and the per-capita contribution are weakly decreasing in group size. ${ }^{12}$ Hence, freeriding tendencies in the literal and in figurative sense (i.e., easy riding in the terminology of Cornes and Sandler, 1996) are exacerbated if more agents enter the economy. In the following, I will show that these findings do not hold in the present model if replications of the whole economy are considered and if the altruists do not only care about aggregate giving, but about the well-being of single individuals.

The altruists' utility functions (3) differ from the utility functions $U^{i}\left(x_{i}, G\right)$ of the public good model in two ways. First, a private motive $g_{i}$ of charitable giving is taken into account. Second, the additional group size parameter $K$ is introduced. Note that the warm-glow effect $g_{i}$ only ameliorates but does not abolish the

\footnotetext{
${ }^{11}$ In fact, for preferences where the rule (8) leads to a unique amount $G^{*}(N, K)$ (see Bergstrom and Cornes (1983) for a characterization of these preferences in the absence of a warmglow effect), any provision level $G$ between $G^{*}(N, K)$ and the altruists' aggregate endowment $N \sum_{i=1}^{I} \omega_{i}$ is Pareto efficient whereas allocations with $G<G^{*}(N, K)$ are Pareto inefficient.

${ }^{12}$ Similar findings can be found in McGuire (1974), Chamberlin (1974), and Andreoni (1988). The paper by Fries et al. (1991) is closest to the present analysis because it also models a change in group size by replicating an initial economy with heterogeneous individuals.
} 
altruists' incentive for free riding if new replicas enter the economy. This means that the results of the literature should also hold in the present model as long as only a partial replication of the economy, namely of the subgroup of altruists, is considered. The following preliminary finding shows that this is indeed the case. The proofs of all propositions are relegated to the Appendix.

Proposition 1: The per-capita contribution $g^{v}(N, K)$ is decreasing and the proportion of contributors $c^{v}(N, K)$ is weakly decreasing in $N$.

Proposition 1 extends the analysis of Fries et al. (1991) by pointing out that $g^{v}(N, K)$ and $c^{v}(N, K)$ are also (weakly) decreasing in the number of potential donors $N I$ if some private, non-altruistic motive $U^{i}\left(\cdot, g_{i}, \cdot\right)$ of charitable giving is taken into account. Clearly, this result also holds for a proportional increase in $N$ and $K$ provided that the functions $U^{i}\left(x_{i}, g_{i}, G, K\right)$ do not depend on $K$. In this case, the altruists care only about the aggregate transfer $G$, such that the number of potential recipients $K$ becomes irrelevant for the amount of charitable giving. In terms of the altruistic preferences (1), this assumption corresponds to the special case $^{13}$

$$
\tilde{U}^{i}\left(x_{i}, g_{i}, \tilde{a}^{K}\left(b_{1}, \ldots, b_{K}\right)\right)=\tilde{U}^{i}\left(x_{i}, g_{i}, \sum_{k=1}^{K} b_{k}\right)
$$

In fact, with preferences of the form (9), the utility functions $U^{i}\left(x_{i}, g_{i}, G, K\right)$ are independent of $K{ }^{14}$ This means that the present model becomes equivalent to the standard specification with utility $U^{i}\left(x_{i}, g_{i}, G\right)$, where a change in $K$ has no effect on charitable giving $G^{v}(N, K)$. Not surprisingly, the same results as in this literature are thus obtained. This observation can be summarized by means of

Corollary 1: Consider altruistic preferences of the form (9). Then $g^{v}(N, K)$ and $c^{v}(N, K)$ do not depend on the number of recipients $K$. Accordingly, $g^{v}(N, N)$ is decreasing and $c^{v}(N, N)$ is weakly decreasing in $N$.

\footnotetext{
${ }^{13}$ Example (9) and the subsequent examples (10), (11), and (13) are expressed in terms of the utility function (1), which forms the starting point of the present investigation. In this way, the reader can verify that these examples do not violate the symmetry assumption (2), which in turn implies that the simpler utility function (3) can indeed be used in the formal analysis.

${ }^{14}$ In example (9), we have $\tilde{a}^{K}\left(b_{1}, \ldots, b_{K}\right)=\sum_{k=1}^{K} b_{k}$, which implies $a^{K}(b, K)=K b$ and $a^{K}(G / K, K)=G$. Hence, the functions $U^{i}(\cdot)$ do not depend on the number of recipients $K$.
} 
As pointed out in the introductory section, the argument that charitable giving is a nonrival commodity from the altruists' perspective led to the idea that altruistic donations can be analyzed in the same way as voluntary donations to a public good. From this perspective, Proposition 1 and Corollary 1 show that the results about group size of the theoretical literature on voluntary public good provision can be interpreted in terms of the present model in two different ways: first, as a thought experiment where only the number of altruists is changed such that an increase in group size simultaneously leads to a higher ratio between the number of altruists and recipients; second, as an implicit assumption concerning altruistic preferences, namely the hypothesis that the donors take only the aggregate transfer $G=\sum_{k=1}^{K} b_{k}$ into account and do not care about the number $K$ of individuals among which these donations have to be allocated.

In the following, an increase in group size is identified with replications of the whole economy. Therefore, I will concentrate on the question whether the findings of Corollary 1 may change if preferences different from those in example (9) are taken into consideration. ${ }^{15}$ Note that a result differing from that in Corollary 1 can only be obtained if an isolated increase in $K$ increases the per-capita contribution $g^{v}(N, K)$ in the Nash equilibrium. A sufficient condition for this property is that each agent's demand for the public good increases in $K$. Due to the first-order conditions (4), the latter property holds if the marginal rates of substitution $m_{i}\left(x_{i}, g_{i}, G, K\right)$ between public and private consumption are increasing in $K$ for all types $i$. However, since a replication of the whole economy increases the percapita contribution $g^{v}(N, K)$ only if the positive effect of $K$ over-compensates the negative effect of $N$, a stronger assumption has to be made. The following result shows that a proportional increase in the number of altruists and recipients weakly increases $g^{v}(N, K)$, as long as the marginal rates of substitution

$$
\hat{m}_{i}\left(x_{i}, g_{i}, b, K\right):=m_{i}\left(x_{i}, g_{i}, b K, K\right)=m_{i}\left(x_{i}, g_{i}, G, K\right)
$$

between private consumption and the per-capita transfer $b$ are weakly increasing in $K$. Note that the definition of $\hat{m}_{i}(\cdot)$ makes use of the relationship $b K=G$.

\footnotetext{
${ }^{15}$ Abrams and Schmitz (1984) provide evidence that the number of potential recipients has a positive effect on charitable giving. This result suggests that other preferences than in (9) are relevant from an empirical point of view.
} 
Hence, the assumption $\hat{m}_{i}\left(x_{i}, g_{i}, b, K+1\right) \geq \hat{m}_{i}\left(x_{i}, g_{i}, b, K\right)$ is stronger than the assumption $m_{i}\left(x_{i}, g_{i}, G, K+1\right) \geq m_{i}\left(x_{i}, g_{i}, G, K\right)$ because the marginal rates of substitution $m_{i}\left(x_{i}, g_{i}, G, K\right)$ are assumed to be decreasing in $G$.

Proposition 2: (a) If the marginal rates of substitution $\hat{m}_{i}\left(x_{i}, g_{i}, b, K\right)$ are independent of $K$ for all types $i \in\{1, \ldots, I\}, g^{v}(N, N)$ and $c^{v}(N, N)$ are independent of $N$. (b) If the marginal rates of substitution $\hat{m}_{i}\left(x_{i}, g_{i}, b, K\right)$ are increasing (decreasing) in $K$ for all types $i \in\{1, \ldots, I\}, g^{v}(N, N)$ is increasing (decreasing) in $N$.

For the intuition behind this finding, consider the case where the functions $\hat{m}_{i}\left(x_{i}, g_{i}, b, K\right)$ are increasing in $K$. Assume now that $K$ and $N$ are increased to $K+1$ and $N+1$ respectively, and that the additional altruist of each type $i$ imitates the existing altruists by choosing the same initial contribution $g_{i}^{v}(N, K)$. This implies that the aggregate donation $G$ increases, whereas $x_{i}, g_{i}$, and $b$ remain constant for all individuals. However, in this situation the contributing types have an incentive to increase their initial contribution $g_{i}^{v}(N, K)>0$ because the change in $K$ has increased their marginal rates of substitution $\hat{m}_{i}\left(x_{i}, g_{i}, b, K\right) \geq 1$ between the per-capita transfer $b$ and 'private' consumption $\left(x_{i}, g_{i}\right)$. Therefore, a full replication of the economy leads to a higher per-capita contribution $g^{v}(N, K)$.

Proposition 2 shows that the per-capita contribution $g^{v}(N, N)$ in the Nash equilibrium is increasing or decreasing in $N$ if each agent's marginal rate of substitution $\hat{m}_{i}(\cdot)$ is increasing or decreasing in $K$. Clearly, these conditions are only sufficient, but not necessary, for the result. It should be noted that this qualification applies to the subsequent findings as well. In fact, the present analysis concentrates on the case where all altruists have similar preferences; it does not deal with situations where $\hat{m}_{i}(\cdot)$ is decreasing in $K$ for some group of altruists and increasing in $K$ for another group of altruists. In such a situation, the effect of a change in group size depends on the ratio between the donations from these groups, which in turn changes with group size such that general results can hardly be obtained.

Proposition 2 relies on three distinct assumptions concerning the effect of the number of recipients $K$ on the marginal rates of substitution $\hat{m}_{i}\left(x_{i}, g_{i}, b, K\right)$. This raises the question of the assumptions concerning the altruistic preferences (1) under which these three cases are obtained. For a clarification of this point, con- 
sider the benchmark case of group-size neutrality, where a full replication of the initial economy has no effect on the per-capita allocation $\left(x_{i}, g_{i}, b\right)$. According to Proposition 2, this property holds if the functions $\hat{m}_{i}\left(x_{i}, g_{i}, b, K\right)$ are independent of $K$. This assumption means that the donors' incentive to transfer money to the recipients does not change with an increase in $K$ as long as all recipients obtain the same transfer $b$ as before. The latter property corresponds to preferences for which an additively separable utility representation

$$
\tilde{U}^{i}\left(x_{i}, g_{i}, \tilde{a}^{K}\left(b_{1}, \ldots, b_{K}\right)\right)=u^{i}\left(x_{i}, g_{i}\right)+\sum_{k=1}^{K} f^{i}\left(b_{k}\right)
$$

exists. In fact, with preferences of the form (10), we get $U^{i}\left(x_{i}, g_{i}, G, K\right)=$ $u^{i}\left(x_{i}, g_{i}\right)+K f^{i}(G / K) .{ }^{16}$ It can easily be verified that these utility functions imply $\hat{m}_{i}\left(x_{i}, g_{i}, b, K+1\right)=\hat{m}_{i}\left(x_{i}, g_{i}, b, K\right)$ for any $K \geq 1$. Because of Proposition 2 , we thus obtain

Corollary 2: Consider altruistic preferences of the form (10). Then $g^{v}(N, N)$ and $c^{v}(N, N)$ are independent of $N$.

For the intuition behind Corollary 2, consider the initial economy where $I$ altruists care about a single recipient $k=1$. With preferences of the form (10), the altruists' marginal rates of substitution $\tilde{U}_{1}^{i} /\left(\tilde{U}_{x}^{i}-\tilde{U}_{g}^{i}\right)$ between the transfer $b_{1}$ and private consumption do not change if a second recipient $k=2$ enters the economy. Hence, their donations remain constant provided that the new altruists behave equivalently by financing the same donation $b_{2}=b_{1}$ for the recipient $k=2$. In contrast to example (9), where the altruists care only about the aggregate transfer $G$, the donations of the new donors thus do not crowd out those of the initial altruists. Therefore, the positive effect of $K$ on the per-capita contribution $g^{v}(N, K)$ precisely cancels out the negative effect of an increase in $N$.

The Corollaries 1 and 2 refer to altruistic preferences (1), where the functions $\hat{m}_{i}\left(x_{i}, g_{i}, b, K\right)$ are decreasing in $K$ or independent of $K$, respectively. In the following, I will show that the marginal rates of substitution $\hat{m}_{i}\left(x_{i}, g_{i}, b, K\right)$ can be increasing in $K$ as well. For an illustration, consider the example discussed in

\footnotetext{
${ }^{16}$ In example (10), we have $\tilde{a}^{K}\left(b_{1}, \ldots, b_{K}\right)=\sum_{k=1}^{K} f^{i}\left(b_{k}\right)$, which implies $a^{K}(b, K)=K f^{i}(b)$ and $a^{K}(G / K, K)=K f^{i}(G / K)$. Therefore, $U^{i}\left(x_{i}, g_{i}, G, K\right)=u^{i}\left(x_{i}, g_{i}\right)+K f^{i}(G / K)$. Note that assumption (6) can be satisfied only if the functions $f^{i}(\cdot)$ are strictly concave. Therefore, example (10) rules out preferences of the form (9).
} 
the introductory section: Assume that the number $T \geq 0$ of those agents who cannot afford the cost $z$ of a medical treatment generates a negative externality upon the altruists. In the absence of charitable giving, $T$ equals the number of recipients $K$. Private donations $G \leq K z$ reduce this number to $T=K-G / z$, which can equivalently be expressed by means of $T=\sum_{k=1}^{K}\left(1-b_{k} / z\right)$. In terms of the altruistic preferences (1), we thus obtain $\tilde{a}^{K}\left(b_{1}, \ldots, b_{K}\right)=\sum_{k=1}^{K}\left(1-b_{k} / z\right)$, where the functions

$$
\tilde{U}^{i}\left(x_{i}, g_{i}, \tilde{a}^{K}\left(b_{1}, \ldots, b_{K}\right)\right)=\tilde{U}^{i}\left(x_{i}, g_{i}, \sum_{k=1}^{K}\left(1-b_{k} / z\right)\right)
$$

are decreasing in $\tilde{a}^{K}(\cdot)$. With preferences of type (11), the marginal rates of substitution $\hat{m}_{i}\left(x_{i}, g_{i}, b, K\right)$ are increasing in $K$. Due to Proposition 2, the percapita contribution $g^{v}(N, N)$ is thus increasing in $N$. Moreover, the special case (11) also implies that the proportion of contributors $c^{v}(N, N)$ is non-decreasing in $N$. These findings are established in the Appendix and can be summarized by means of

Corollary 3: Consider altruistic preferences of the form (11). Then $g^{v}(N, N)$ is increasing and $c^{v}(N, N)$ is weakly increasing in $N$.

For the intuition behind Corollary 3 , consider again a situation where $K, N$, and $G$ are increased proportionally such that the average transfer $b$ does not change. This implies that the percentage $T / K=1-b / z$ of those individuals who remain without treatment is kept constant. Hence, the absolute amount $T$ of these agents is increased. Since the negative externality depends on $T$, the altruists thus have an incentive to further increase their donation. Therefore, the per-capita contribution and the proportion of contributors among the altruists are (weakly) increasing if the whole economy is replicated.

Example (11) serves as an illustration for Proposition 2, where it is shown that the per-capita contribution $g^{v}(N, N)$ is increasing in $N$ if the altruists' marginal rates of substitution $\hat{m}_{i}(\cdot)$ are increasing in $K$. This condition can be expressed in a more intuitive way if one restricts attention to additively separable utility functions

$$
U^{i}\left(x_{i}, g_{i}, G, K\right)=u^{i}\left(x_{i}, g_{i}\right)+a^{K}(G / K, K),
$$

which imply that the marginal utility of (altruistic) charitable giving $U_{G}^{i}$ depends only on $G$ and $K$, and that $U_{x}^{i}$ and $U_{g}^{i}$ depend only on $x_{i}$ and $g_{i}$. Let $\epsilon_{G}^{i}$ and 
$\epsilon_{K}^{i}$ denote the elasticities of the function $U_{G}^{i}(G, K)$ with respect to $G$ and $K$ respectively. It can easily be verified by means of (12) that the marginal rate of substitution $\hat{m}_{i}(\cdot)$ is increasing in $K$ if and only if $\epsilon_{K}^{i}+\epsilon_{G}^{i}>0$. Hence, the per-capita contribution $g^{v}(N, N)$ is increasing in $N$ if a one-percent increase in the number of recipients $K$ increases the donors' marginal utility from charitable giving to a larger extent than it is decreased by a one-percent increase in charitable giving $G$. If this condition holds, the donors become more inclined towards charitable giving as the number of recipients is increased, even if the per-capita transfer does not change. Note that example (11) - where the altruists care about the number of agents who cannot afford a certain standard of medical treatment (or of other needs like nutrition, shelter and education) - is just one possibility for illustrating such a situation. In fact, based on the elasticity argument presented above, other examples can easily be constructed. ${ }^{17}$

\section{The index of easy riding}

The three examples which have been discussed in Section 3 illustrate different assumptions concerning the effect of a change in the number of recipients $K$ on the altruists' preferences for private consumption and charitable giving. Clearly, this effect is relevant also for the relationship between group size and the efficient level of charitable giving. Therefore, the results of Section 3 do not clarify whether the deficiencies of the Nash equilibrium relative to an efficient allocation become more or less severe as group size is increased. In the following, this issue will be discussed.

In the present context, inefficiency of the Nash equilibrium means that all altruists and all recipients can be made better off if charitable giving is increased above the equilibrium level $G^{v}(N, K)$. This property corresponds to the observation that the efficient provision level $G^{*}(N, K)$ in the Lindahl equilibrium exceeds $G^{v}(N, K)$ for economies with at least two altruists. ${ }^{18}$ We thus

\footnotetext{
${ }^{17}$ Consider, for example, the function $a^{K}(G / K, K)=(G / K)^{\alpha} K^{\beta}$. Assuming (12) and $0<$ $\alpha<1$, it can be shown that $\hat{m}^{i}(\cdot)$ is increasing in $K$ (i.e., that $\epsilon_{K}^{i}+\epsilon_{G}^{i}>0$ ) if and only if $\beta>1$.

18 As shown in Buchholz and Peters (2001) for the voluntary contribution model of public good provision (i.e., for preferences $U^{i}\left(x_{i}, G\right)$ ), efficient provision $G^{*}(\cdot)$ in the Lindahl equi-
} 
have $G^{v}(\cdot) / G^{*}(\cdot)<1$, provided that $N I \geq 2$. This property is equivalent to $g^{v}(\cdot) / g^{*}(\cdot)<1$, where $g^{*}(N, K):=G^{*}(N, K) /(N I)$ is the altruists' per-capita donation in the Lindahl equilibrium. In the theoretical and experimental literature on the link between group size and public good provision, the ratio

$$
e(N, K):=\frac{g^{v}(N, K)}{g^{*}(N, K)}
$$

is usually employed as a measure of underprovision. Following the terminology of Cornes and Sandler $(1984,1996)$, I will refer to this ratio as the index of easy riding. The subsequent analysis investigates how full replications of the initial economy affect $e(N, K)$ for the case of altruistic charitable giving.

In the voluntary contribution model of public good provision where utility functions $U^{i}\left(x_{i}, G\right)$ are assumed, group size and free riding tendencies are positively correlated (see Proposition 1). Hence, intuition suggests that the index of easy riding should decrease in this model as the economy becomes larger. However, neither a general proof of this claim nor a counterexample is available. Therefore, the literature has concentrated on examples where $U^{i}\left(x_{i}, G\right)$ is either quasilinear or Cobb-Douglas (see Sandler 1992, Chapter 7, for a critical discussion). The Cobb-Douglas example has been generalized in Gaube (2001) by showing that the index of easy riding decreases in group size, provided that the commodities $x_{i}$ and $G$ are strictly normal and weak gross substitutes. Since the assumption of strict normality is crucial for uniqueness of the Nash equilibrium and has also been employed in Section 3, I will use this finding as a reference point for the subsequent analysis.

Before investigating the ratio $g^{v}(\cdot) / g^{*}(\cdot)$, consider first the effect of an isolated increase in $N$ and a proportional increase in $N$ and $K$ on the efficient per-capita donation $g^{*}(N, K)$ : The commodities $x_{i}$ and $G$ are weak gross substitutes for an agent of type $i$, as long as the demand function $x_{i}\left(p_{i}, \omega_{i}, K\right)$ in the Lindahl economy is non-decreasing in the personalized price $p_{i}$ of the public good. In analogy to Lemma 2 of Gaube (2001), part (a) of the following Proposition 3 points out that this assumption is sufficient for obtaining a positive relationship between the per-capita contribution $g^{*}(N, K)$ in the Lindahl equilibrium and

librium always exceeds the equilibrium provision $G^{v}(\cdot)$. Using the same argument as in their paper, it can be shown that $G^{*}(N, K)>G^{v}(N, K)$ holds in the present context as well. 
the group size parameter $N$. In contrast, part (b) of Proposition 3 refers to a proportional increase in $N$ and $K$. Similar to Proposition 2, it is shown that $g^{*}(N, N)$ is increasing in $N$, as long as an additional recipient has a non-negative effect on the marginal rates of substitution $\hat{m}_{i}\left(x_{i}, g_{i}, b, K\right)$.

Proposition 3: (a) If public and private consumption are weak gross substitutes for all types $i \in\{1, \ldots, I\}, g^{*}(N, K)$ is weakly increasing in $N$.

(b) If the functions $\hat{m}_{i}\left(x_{i}, g_{i}, b, K\right)$ are weakly increasing in $K$ for all types $i \in$ $\{1, \ldots, I\}, g^{*}(N, N)$ is increasing in $N$.

Consider first part (a) of Proposition 3. The following intuition lies behind this finding: Since an increase in the number of altruists decreases the Lindahl prices $p_{i}^{*}(N, K)$, the individuals' contributions $p_{i} G=\omega_{i}-x_{i}$ are weakly increasing in $N$, as long as the functions $x_{i}\left(p_{i}, \omega_{i}, K\right)$ are weakly increasing in $p_{i}$. Because of Proposition 1, the gross-substitutes assumption thus implies that the index of easy riding $e(N, K)$ is decreasing in $N$. Clearly, this finding also holds for a proportional increase in $N$ and $K$, provided that an isolated change of $K$ does not affect $g^{*}(N, K)$ and $g^{v}(N, K)$ respectively. The latter property holds if the utility functions $U^{i}\left(x_{i}, g_{i}, G, K\right)$ are independent of $K$, i.e., if preferences can be described by means of example (9). In this case, Corollary 1 and Proposition 3 thus imply

Corollary 4: Consider altruistic preferences of the form (9) and assume that public and private consumption are weak gross substitutes for all types $i=1, \ldots, I$. Then the index of easy riding $e(N, N)$ is decreasing in $N$.

With altruistic preferences of type (9), the present model is equivalent to the public good model $U^{i}\left(x_{i}, G\right)$ because a replication of the group of altruists has the same effect on charitable giving as a replication of the whole economy. In analogy to Corollary 1, Corollary 4 thus serves as a reference point for investigating the potential differences between non-altruistic public good provision and an altruistic motive for charitable giving. For that purpose, I will compare Corollary 4 with examples (10) and (11) of Section 3 where, in contrast to example (9), an increase in the number of recipients $K$ has a positive effect on the equilibrium provision level $g^{v}(N, K)$ and the efficient provision level $g^{*}(N, K)$.

Consider first example (10). Part (b) of Proposition 3 points out that $g^{*}(N, N)$ 
is increasing in $N$ if the effect of $K$ on the marginal rates of substitution $m_{i}\left(x_{i}, g_{i}, G, K\right)$ is sufficiently strong such that the functions $\hat{m}_{i}\left(x_{i}, g_{i}, b, K\right)$ are weakly increasing in $K$ as well. Combining this result with part (a) of Proposition 2 , it becomes clear that the ratio $g^{v}(N, N) / g^{*}(N, N)$ is decreasing in $N$, as long as the marginal rates of substitution $\hat{m}_{i}\left(x_{i}, g_{i}, b, K\right)$ are independent of $K$. Since this property holds as long as altruistic preferences are of the additively separable form (10), we can state

Corollary 5: Consider altruistic preferences of the form (10). Then $e(N, N)$ is decreasing in $N$.

The per-capita donations $g^{v}(N, K)$ and $g^{*}(N, K)$ are increasing in $K$ for the preferences (10), but are independent of $K$ for the preferences (9). The comparison between these examples thus shows that a positive effect of $K$ on charitable giving $g^{v}(N, K)$ does not imply a lower degree of underprovision relative to the efficient level $g^{*}(N, K)$ because the latter increases in $K$ as well. In fact, since Corollary 5 also holds if the gross-substitutes assumption is violated, example (10) makes a stronger point than example (9) for the claim that the efficiency measure $e(N, N)$ is decreasing in group size. Still, the subsequent analysis points out that group size and the index of easy riding can also be positively correlated in the present context. This will be illustrated by means of example (11).

In examples (9) and (10) the marginal rates of substitution $\hat{m}_{i}\left(x_{i}, g_{i}, b, K\right)$ are non-increasing in $K$. In these examples, underprovision becomes more severe as group size increases. Consider now a situation where the functions $\hat{m}_{i}\left(x_{i}, g_{i}, b, K\right)$ are increasing in $K$. According to Propositions 2 and 3, this implies that $g^{v}(N, N)$ and $g^{*}(N, N)$ are increasing in $N$. Note that $g^{*}(N, N)$ cannot exceed the altruists' average endowment $\bar{\omega}:=\left(\sum_{i=1}^{I} \omega_{i}\right) / I$, which means that is has to converge to some upper bound $\bar{g} \leq \bar{\omega}$. Hence, as long as the positive effect of $N$ on the equilibrium level $g^{v}(N, N)$ is not arbitrarily small, the index of easy riding $e(N, N)$ must increase in group size at least for some $N$. The following proposition shows that $e(N, N)$ can even converge to unity if the positive effect of $K$ on the marginal rates of substitution $\hat{m}_{i}\left(x_{i}, g_{i}, b, K\right)$ does not vanish as $K$ converges to infinity. This is formalized by means of the assumption that one can always find some number $\tilde{K} \geq 1$ such that $\hat{m}_{i}\left(\bar{x}_{i}, \bar{g}_{i}, \bar{b}, \tilde{K}\right)$ exceeds unity provided that $\hat{m}_{i}\left(\bar{x}_{i}, \bar{g}_{i}, \bar{b}, 1\right)$ is positive, and provided that the allocation $\left(\bar{x}_{i}, \bar{g}_{i}, \bar{b}\right)$ is consistent 
with the altruists' budget constraints. Intuitively, this assumption means that, for a constant per-capita transfer $b$, an increase in $K$ does not only increase the marginal utility of charitable giving $U_{G}^{i}$, but that this effect is in principle unbounded, i.e., that $U_{G}^{i}$ grows without bounds as $K$ converges to infinity. Under this assumption, the donors become gradually more inclined towards voluntary charitable giving as group size is increased, such that the difference between voluntary donations $G^{v}(N, N)$ and efficient donations $G^{*}(N, N)$ remains finite in a large economy. Accordingly, the difference between the per-capita donations $g^{v}(N, N)$ and $g^{*}(N, N)$ becomes arbitrarily small as $N$ converges to infinity.

Proposition 4: Assume that the functions $\hat{m}_{i}\left(x_{i}, g_{i}, b, K\right)$ are increasing in $K$ for all types $i \in\{1, \ldots, I\}$ and that $\lim _{K \rightarrow \infty} \hat{m}_{i}\left(\bar{x}_{i}, \bar{g}_{i}, \bar{b}, K\right)>1$ holds for all $\left(\bar{x}_{i}, \bar{g}_{i}, \bar{b}\right)$ which satisfy the condition $0<\hat{m}_{i}\left(\bar{x}_{i}, \bar{g}_{i}, \bar{b}, \bar{K}\right) \leq 1$, where $\bar{x}_{i}+\bar{g}_{i}=\omega_{i}$ and $\bar{K}=1$. Then $e(N, N)$ is increasing in $N$ at least for some $N \geq 1$, and it converges to unity as $N$ converges to infinity.

The following formal reasoning is behind Proposition 4: Consider the case $I=$ 1 , which means that all altruists are identical and that their Lindahl prices equal $1 / N$. Hence, an allocation $\left(\bar{x}_{i}, \bar{g}_{i}, \bar{b}\right)$ is the Lindahl equilibrium of an economy with parameters $\bar{N}=\bar{K} \geq 2$, provided that $\hat{m}_{i}\left(\bar{x}_{i}, \bar{g}_{i}, \bar{b}, \bar{K}\right)=1 / \bar{N}$. Because of the first-order conditions (4), the same vector $\left(\bar{x}_{i}, \bar{g}_{i}, \bar{b}\right)$ is also a Nash equilibrium in an economy with parameters $\tilde{N}=\tilde{K}$, as long as $\hat{m}_{i}\left(\bar{x}_{i}, \bar{g}_{i}, \bar{b}, \tilde{K}\right)=1$. The assumption $\lim _{K \rightarrow \infty} \hat{m}_{i}\left(\bar{x}_{i}, \bar{g}_{i}, \bar{b}, K\right)>1$ of Proposition 4 thus implies that any allocation $\left(\bar{x}_{i}, \bar{g}_{i}, \bar{b}\right)$ that is efficient in the $\bar{N}$ - $\bar{K}$-economy can be implemented as a Nash equilibrium if the corresponding group size $\tilde{K}=\tilde{N}$ becomes sufficiently large. Therefore, $g^{v}(N, N)$ and $g^{*}(N, N)$ must converge to the same upper bound $\bar{g} \leq \bar{\omega}$ as group size $N$ converges to infinity. Accordingly, the index of easy riding $e(N, N)$ converges to unity, which means that it cannot decrease in group size for any $N \geq 1$.

For an example of preferences which are in line with the assumptions of Proposition 4 , consider a special case of the utility functions (11), namely

$$
\tilde{U}^{i}\left(x_{i}, g_{i}, \tilde{a}^{K}\left(b_{1}, \ldots, b_{K}\right)\right)=u^{i}\left(x_{i}, g_{i}\right)-\left(\sum_{k=1}^{K}\left(1-b_{k} / z\right)\right)^{\alpha}, \quad \alpha>1 .
$$

Because of $\sum_{k=1}^{K}\left(1-b_{k} / z\right)=K-G / z$, example (13) implies $U^{i}\left(x_{i}, g_{i}, G, K\right)=$ $u^{i}\left(x_{i}, g_{i}\right)-(K-G / z)^{\alpha}$. It can easily be verified that this functional form satisfies 
the assumptions of Proposition $4 .^{19}$ We can thus state

Corollary 6: Consider altruistic preferences of the form (13). Then $e(N, N)$ is increasing in $N$ at least for some $N \geq 1$, and it converges to unity as $N$ converges to infinity.

For the intuition behind Corollary 6, consider the interpretation of example (11) in Section 3, where the term $T=K-G / z$ stands for the number of agents who remain without medical treatment. In example (13), this number affects the marginal utility of charitable giving by means of the formula $U_{G}^{i}=(\alpha / z) T^{\alpha-1}$. The important point about this formula is that it converges to infinity as $T$ converges to infinity. Assume now for simplicity that the altruists' endowments are sufficiently high relative to the per-capita cost of medical treatment $z$ such that the donors' marginal utility from private consumption $U_{x}^{i}$ is finite even if aggregate donations cover the maximum total cost $z K .{ }^{20}$ Then the marginal utility $U_{G}^{i}$ and the number $T$ must be finite in the Nash equilibrium as well. In other words, since the altruists suffer from $T$, voluntary donations keep this number finite even if the number of potential recipients grows to infinity. Accordingly, the difference between equilibrium provision $G^{v}(N, N)$ and efficient provision $G^{*}(N, N)$ must also be finite, which in turn implies that the difference between the per-capita contributions $g^{*}(N, N)$ and $g^{v}(N, N)$ converges to zero as group size converges to infinity.

Proposition 4 and Corollary 6 stand in stark contrast to the widespread claim that the free-rider problem of voluntary charitable giving becomes more severe as group size is increased. Note, however, that the findings rely on a thought experiment where it is assumed that the economy's size $N=K$ grows to infinity. This raises the question whether a positive relationship between group size and the index of easy riding $e(N, N)$ can also be established for a small group of agents. In order to show that this is indeed the case, consider an economy with a single

\footnotetext{
${ }^{19}$ The utility function $U^{i}\left(x_{i}, g_{i}, G, K\right)=u^{i}\left(x_{i}, g_{i}\right)-(K-G / z)^{\alpha}$ leads to $m_{i}\left(x_{i}, g_{i}, G, K\right)=$ $\alpha\left[(K-G / z)^{\alpha-1} /\left(u_{x}^{i}-u_{g}^{i}\right)\right]$, which in turn implies $\hat{m}_{i}\left(x_{i}, g_{i}, b, K\right)=\alpha K^{\alpha-1}\left[(1-b / z)^{\alpha-1} /\left(u_{x}^{i}-\right.\right.$ $\left.\left.u_{g}^{i}\right)\right]$. Hence, $\hat{m}_{i}\left(x_{i}, g_{i}, b, K\right)$ is increasing in $K$ and exceeds unity for any vector $\left(\bar{x}_{i}, \bar{g}_{i}, \bar{b}\right)$, as defined in Proposition 4, provided that $K$ becomes sufficiently large.

${ }^{20}$ Note that Corollary 6 does not rely on this assumption. It is used here only for clarifying the main argument behind the result.
} 
type of donor, i.e., $I=1$. This assumption means that we have only one altruist in the initial economy of size $N=K=1$. Since no positive externality among the altruists can be generated in this case, an efficient allocation is obtained such that $e(1,1)=1$. With at least two altruists, however, voluntary contributions are inefficiently low (see fn. 18). Therefore, the inequality $e(2,2)<e(1,1)$ holds generally with a single type of donor. Assume now that the altruists' preferences can be described by a utility function of the form (13), and consider the example $u^{i}\left(x_{i}, g_{i}\right)=\ln x_{i}+\delta \ln g_{i}$, where $\delta \geq 0$. A numerical analysis of this example shows that the graph of the function $e(N, N)$ is U-shaped, i.e., that $e(N, N)$ decreases in $N$ for all $N$ below some threshold $\bar{N} \geq 2$ and increases in $N$ for all $N \geq \bar{N} \cdot{ }^{21}$ Depending on the parameters $\alpha, \delta, z$ and the donors' endowment $\omega_{i}$, any threshold value $\bar{N} \geq 2$ can be generated. Hence, the index of easy riding $e(N, N)$ can increase in $N$, even for small groups with $N \geq 3$ altruists and recipients.

\section{Conclusion}

The present paper relies on the presumption that the comparison between charitable giving in a small and a large community should take into account that the same group structure is investigated in both cases only if the number of potential donors and the number of potential recipients is changed proportionally. Therefore, a model is introduced where altruistic motives are expressed in terms of utility interdependence, such that the effect of an increase in the size of each subgroup can be analyzed. It is shown that this model is equivalent to the voluntary contribution model of public good provision, provided that a specific structure of altruistic preferences is assumed. In this case, the main results of the literature are confirmed, namely that an increase in group size leads to a decrease in (i) per-capita contributions, (ii) the ratio between contributors and free riders, and (iii) the ratio between the equilibrium level and the efficient level of public good

\footnotetext{
${ }^{21}$ The argument presented above makes it clear that the index of easy riding must decrease in group size at least for the smallest possible group with a single altruist. Under the assumptions of Proposition 4, the index must also increase in group size for very large groups. Note, however, that these two findings do not imply that the function $e(N, N)$ is $\mathrm{U}$-shaped. The numerical example discussed in the text thus illustrates a possible functional form, which by no means follows from the assumptions made in Proposition 4.
} 
provision. However, if the altruists care not just about the aggregate donation, but also about the number of potential recipients, each of these variables can be positively correlated with group size. Hence, the free rider problem of voluntary charitable giving may not become worse as group size is increased because monetary transfers are equivalent to a public good only from the altruists', but not from the recipients' perspective.

The model presented in Section 2 provides a theoretical framework for analyzing the link between group size and charitable giving, which does not presume a specific functional form of altruistic preferences from the outset. In this way, it is pointed out by means of examples (9) - (13) that the form of altruistic preferences is crucial for answering the question whether an increase in group size alleviates or exacerbates the free-rider problem of charitable giving. However, the analysis does not clarify which type of preferences is most relevant from an empirical point of view. To my knowledge, neither empirical nor experimental results are available that refer to the effect of group size on purely altruistic donations, i.e., voluntary contributions where, like in the dictator game, the donors' private interest in consuming the aggregate donation is ruled out by assumption. ${ }^{22}$ Therefore, further research is required in order to shed light on the question whether real-world altruistic preferences imply that private charitable giving becomes more (or less) efficient as group size is increased.

\section{Appendix}

\section{Proof of Proposition 1}

Consider the Nash equilibrium in two economies with parameters $(\tilde{N}, K)$ and $(\bar{N}, K)$, respectively where $\bar{N}=\tilde{N}+1$ and $K \geq 1$. In order to simplify the notation, define $\tilde{g}_{i}:=g_{i}^{v}(\tilde{N}, K), \tilde{x}_{i}:=\omega_{i}-\tilde{g}_{i}, \tilde{g}:=g^{v}(\tilde{N}, K)$, and $\tilde{G}:=G^{v}(\tilde{N}, K)$. The equilibrium allocation $\bar{g}_{i}, \bar{x}_{i}, \bar{g}, \bar{G}$ for the $\bar{N}$ - $K$-economy is defined analogously. Assume now that $g^{v}(N, K)$ is not decreasing in $N$, i.e., that $\bar{g} \geq \tilde{g}$. This implies $\bar{G}>\tilde{G}$. Due to assumption (6), we thus have $m_{i}\left(\tilde{x}_{i}, \tilde{g}_{i}, \bar{G}, K\right)<m_{i}\left(\tilde{x}_{i}, \tilde{g}_{i}, \tilde{G}, K\right)$.

\footnotetext{
${ }^{22}$ In fact, the different cases analyzed in Sections 3 and 4 could be investigated experimentally by comparing the donations in (multiperson) dictator games with different numbers of potential donors and recipients.
} 
Because of the first-order conditions (4), this implies $\bar{g}_{i}=\tilde{g}_{i}=0$ for all types $i \notin C^{v}(\tilde{N}, K)$ and $\bar{g}_{i} \leq \tilde{g}_{i}$ for all types $i \in C^{v}(\tilde{N}, K)$, where the strict inequality holds provided that $0<\tilde{g}_{i}<\omega_{i}$. Due to assumption (7), we thus have $\bar{g}<\tilde{g}$, which contradicts the initial claim $\bar{g} \geq \tilde{g}$. Therefore, $g^{v}(N, K)$ is decreasing in $N$.

The result $\bar{g}<\tilde{g}$ implies $\bar{g}_{j}<\tilde{g}_{j}$ for at least some type $j \in\{1, \ldots, I\}$. Because of the first-order conditions (4), this can hold only if $m_{j}\left(\tilde{x}_{j}, \tilde{g}_{j}, \bar{G}, K\right)<$ $m_{j}\left(\tilde{x}_{j}, \tilde{g}_{j}, \tilde{G}, K\right)$. Since $m_{j}(\cdot)$ is decreasing in $G$, we thus have $\bar{G}>\tilde{G}$. Using the first-order conditions (4) for the types $i \neq j$ as well, the property $\bar{G}>\tilde{G}$ leads to $\bar{g}_{i} \leq \tilde{g}_{i}$ for all $i \neq j$. Therefore, $c^{v}(N, K)$ is weakly decreasing in $N$.

\section{Proof of Proposition 2}

Consider the Nash equilibrium in two economies with parameters $\tilde{N}=\tilde{K}$ and $\bar{N}=\bar{K}=\tilde{N}+1$ respectively. In order to simplify the notation, define $\tilde{g}_{i}:=$ $g_{i}^{v}(\tilde{N}, \tilde{N}), \tilde{x}_{i}:=\omega_{i}-\tilde{g}_{i}, \tilde{g}:=g^{v}(\tilde{N}, \tilde{N}), \tilde{G}:=G^{v}(\tilde{N}, \tilde{N})$, and $\tilde{b}:=\tilde{G} / \tilde{K}$. The equilibrium allocation $\bar{g}_{i}, \bar{x}_{i}, \bar{g}, \bar{G}, \bar{b}$ for the $\bar{N}$ - $\bar{K}$-economy is defined analogously.

(a) Note that $\bar{g}_{i}=\tilde{g}_{i}, i=1, \ldots, I$ implies $\bar{x}_{i}=\tilde{x}_{i}, \bar{g}=\tilde{g}$, and $\bar{b}=$ $\tilde{b}$. Since it is assumed that the functions $\hat{m}_{i}(\cdot)$ do not depend on $K$, we also have $\hat{m}_{i}\left(\tilde{x}_{i}, \tilde{g}_{i}, \tilde{b}, \bar{K}\right)=\hat{m}_{i}\left(\tilde{x}_{i}, \tilde{g}_{i}, \tilde{b}, \tilde{K}\right)$. Hence, the first-order conditions $\hat{m}_{i}\left(\tilde{x}_{i}, \tilde{g}_{i}, \tilde{b}, \tilde{K}\right)=m_{i}\left(\tilde{x}_{i}, \tilde{g}_{i}, \tilde{G}, \tilde{K}\right) \geq(\leq) 1$ for the contributors (non-contributors) in the $\tilde{N}$ - $\tilde{K}$-economy hold for the $\bar{N}$ - $\bar{K}$-economy as well. The vector $\left(\tilde{g}_{1}, \tilde{g}_{2}, \ldots, \tilde{g}_{I}\right)$ is thus a Nash equilibrium in both economies. Therefore, $g^{v}(N, N)$ and $c^{v}(N, N)$ are independent of $N$.

(b) In the following, I will only show that $\bar{g}>\tilde{g}$ holds as long as the functions $\hat{m}_{i}\left(x_{i}, g_{i}, b, K\right)$ are increasing in $K$. The proof of the opposite case is equivalent. Note first that we have $\partial \hat{m}_{i}(\cdot) / \partial x_{i}=\partial m_{i}(\cdot) / \partial x_{i}, \partial \hat{m}_{i}(\cdot) / \partial g_{i}=\partial m_{i}(\cdot) / \partial g_{i}$, and $\partial \hat{m}_{i}(\cdot) / \partial b=\left(\partial m_{i}(\cdot) / \partial G\right) K$. Therefore, assumption (6) is equivalent to the assumption that $\hat{m}_{i}(\cdot)$ is increasing in $x_{i}$, decreasing in $b$, and weakly decreasing in $g_{i}$. Assume now that the claim $\bar{g}>\tilde{g}$ is not correct, i.e., that $\bar{g} \leq \tilde{g}$ holds in equilibrium. Because $\bar{N} / \bar{K}=\tilde{N} / \tilde{K}$, this implies $\bar{b} \leq \tilde{b}$. Since $\hat{m}_{i}(\cdot)$ is increasing in $K$ and decreasing in $b$, we thus have $\bar{m}_{i}:=\hat{m}_{i}\left(\tilde{x}_{i}, \tilde{g}_{i}, \bar{b}, \bar{K}\right)>\hat{m}_{i}\left(\tilde{x}_{i}, \tilde{g}_{i}, \tilde{b}, \tilde{K}\right):=\tilde{m}_{i}$. Note that $\tilde{m}_{i} \geq 1$ holds for all types $i \in C^{v}(\tilde{N}, \tilde{N})$. Therefore, $\bar{m}_{i}>1$ for all $i \in C^{v}(\tilde{N}, \tilde{N})$, which in turn implies $\bar{g}_{i}>\tilde{g}_{i}$, as long as $0<\tilde{g}_{i}<\omega_{i}$, and $\bar{g}_{i}=\tilde{g}_{i}$, as long as $\tilde{g}_{i}=\omega_{i}$. Because of assumption (7), we thus obtain $\bar{g}>\tilde{g}$, which 
contradicts the initial claim $\bar{g} \leq \tilde{g}$. Hence, $g^{v}(N, N)$ is increasing in $N$.

\section{Proof of Corollary 3}

Because of $\tilde{a}^{K}\left(b_{1}, \ldots, b_{K}\right)=\sum_{k=1}^{K}\left(1-b_{k} / z\right)$, we have $a^{K}(b, K)=K(1-b / z)$, $a^{K}(G / K, K)=K-G / z$, and $U^{i}\left(x_{i}, g_{i}, G, K\right)=\tilde{U}^{i}\left(x_{i}, g_{i}, K-G / z\right)$. Accordingly, $U_{G}^{i}=\tilde{U}_{a}^{i}(-1 / z), m_{i}\left(x_{i}, g_{i}, G, K\right)=\tilde{m}_{i}\left(x_{i}, g_{i}, a^{K}(G / K, K)\right)(-1 / z)$, and $\hat{m}_{i}\left(x_{i}, g_{i}, b, K\right)=\tilde{m}_{i}\left(x_{i}, g_{i}, a^{K}(b, K)\right)(-1 / z)$, where $\tilde{m}_{i}\left(x_{i}, g_{i}, a^{K}(b, K)\right):=$ $\left(\tilde{U}_{a}^{i} /\left(\tilde{U}_{x}^{i}-\tilde{U}_{g}^{i}\right)\right)$. Hence, $b$ and $K$ affect $\hat{m}_{i}\left(x_{i}, g_{i}, b, K\right)$ only by means of the term $a^{K}(b, K)=K(1-b / z)$, which is decreasing in $b$ and increasing in $K$. Since the assumption $\partial m_{i}(\cdot) / \partial G<0$ in (6) is equivalent to $\partial \hat{m}_{i}(\cdot) / \partial b<0$, the functions $\hat{m}_{i}\left(x_{i}, g_{i}, b, K\right)$ are thus increasing in $K$. Due to Proposition 2, this implies that $g^{v}(N, N)$ is increasing in $N$.

Consider now the Nash equilibrium in two economies with parameters $\tilde{N}=\tilde{K}$ and $\bar{N}=\bar{K}=\tilde{N}+1$ respectively, and employ the same notation as in the proof of Proposition 2. Because of $\bar{g}>\tilde{g}$, we must have $\bar{g}_{j}>\tilde{g}_{j}$ for at least one type $j \in\{1, \ldots, I\}$. Due to the agents' first-order conditions (4), this can hold only if $\hat{m}_{j}\left(\tilde{x}_{j}, \tilde{g}_{j}, \bar{b}, \bar{K}\right)>\hat{m}_{j}\left(\tilde{x}_{j}, \tilde{g}_{j}, \tilde{b}, \tilde{K}\right)$. In the present example, this leads to $\tilde{m}_{j}\left(\tilde{x}_{j}, \tilde{g}_{j}, \bar{a}\right)>\tilde{m}_{j}\left(\tilde{x}_{j}, \tilde{g}_{j}, \tilde{a}\right)$, where $\bar{a}:=a^{\bar{K}}(\bar{b}, \bar{K})$ and $\tilde{a}:=a^{\tilde{K}}(\tilde{b}, \tilde{K})$. Since the property $\partial \hat{m}_{i}(\cdot) / \partial b<0$ implies that $\tilde{m}_{i}(\cdot)$ is decreasing in $a^{K}(b, K)$, we thus have $\bar{a}<\tilde{a}$. Accordingly, $\hat{m}_{i}\left(\tilde{x}_{i}, \tilde{g}_{i}, \bar{b}, \bar{K}\right)>\hat{m}_{i}\left(\tilde{x}_{i}, \tilde{g}_{i}, \tilde{b}, \tilde{K}\right)$ holds for all types $i \in\{1, \ldots, I\}$. Using again the first-order conditions (4), this implies $\bar{g}_{i} \geq \tilde{g}_{i}$ for all $i$. Therefore, $c^{v}(\bar{N}, \bar{N}) \geq c^{v}(\tilde{N}, \tilde{N})$.

\section{Proof of Proposition 3}

Consider first the individuals' first-order conditions $m_{i}\left(x_{i}, g_{i}, G, K\right)=p_{i}$ in the Lindahl equilibrium, where $g_{i}=p_{i} G$ and $x_{i}=\omega_{i}-g_{i}$. Because $\partial m_{i}(\cdot) / \partial x_{i}>0$, $\partial m_{i}(\cdot) / \partial G<0$, and $\partial m_{i}(\cdot) / \partial g_{i} \leq 0$, these conditions imply that the demand functions $G_{i}\left(p_{i}, \omega_{i}, K\right)$ are strictly decreasing in $p_{i}$. For the same reason, $G_{i}\left(p_{i}, \omega_{i}, K\right)$ is increasing in $K$, provided that $m_{i}\left(x_{i}, g_{i}, G, K\right)$ is increasing in $K$.

(a) Let define $x_{i}^{*}(N, K):=x_{i}\left(p_{i}^{*}(N, K), \omega_{i}, K\right)$ and $g_{i}^{*}(N, K):=\omega_{i}-x_{i}^{*}(N, K)$. Since the functions $G_{i}\left(p_{i}, \omega_{i}, K\right)$ are strictly decreasing in $p_{i}$, the equilibrium conditions $N\left(\sum_{i=1}^{I} p_{i}\right)=1$ and $G_{1}(\cdot)=G_{2}(\cdot)=\ldots=G_{I}(\cdot)$ imply $p_{i}^{*}(N+1, K)<$ 
$p_{i}^{*}(N, K)$ for all types $i=1, \ldots, I$. Because of weak gross substitutability, we thus have $x_{i}^{*}(N+1, K) \leq x_{i}^{*}(N, K)$ for all $i$, which in turn implies $g_{i}^{*}(N+1, K) \geq$ $g_{i}^{*}(N, K)$. Accordingly, $g^{*}(N+1, K) \geq g^{*}(N, K)$ holds as well.

(b) Consider the Lindahl equilibrium in two economies with parameters $\tilde{N}=\tilde{K}$ and $\bar{N}=\bar{K}=\tilde{N}+1$ respectively. In order to simplify the notation, define $\tilde{x}_{i}:=x_{i}^{*}(\tilde{N}, \tilde{N}), \tilde{g}_{i}:=g_{i}^{*}(\tilde{N}, \tilde{N}), \tilde{p}_{i}:=p_{i}^{*}(\tilde{N}, \tilde{N}), \tilde{G}:=G^{*}(\tilde{N}, \tilde{N})$, $\tilde{g}:=g^{*}(\tilde{N}, \tilde{N})$, and $\tilde{b}:=\tilde{G} / \tilde{K}$. The equilibrium prices $\bar{p}_{i}$ and allocation $\bar{x}_{i}, \bar{g}_{i}, \bar{G}, \bar{g}, \bar{b}$ for the $\bar{N}$ - $\bar{K}$-economy are defined analogously. Note first that the definition $\hat{m}_{i}\left(x_{i}, g_{i}, b, K\right):=m_{i}\left(x_{i}, g_{i}, b K, K\right)=m_{i}\left(x_{i}, g_{i}, G, K\right)$ and the assumption $\partial m_{i}(\cdot) / \partial G<0$ imply that $\hat{m}_{i}(\cdot)$ can be weakly increasing in $K$ only if $m_{i}(\cdot)$ is strictly increasing in $K$. Therefore, the functions $G_{i}\left(p_{i}, \omega_{i}, K\right)$ are increasing in $K$ and decreasing in $p_{i}$ for all types $i$ (see above). Note also that the equilibrium condition $N \sum_{i=1}^{I} p_{i}(N, N)=1$ implies $\bar{p}_{i}<\tilde{p}_{i}$ for at least one type $i \in\{1, \ldots, I\}$. Therefore, we have $\bar{G}=G_{i}\left(\bar{p}_{i}, \omega_{i}, \bar{K}\right)>G_{i}\left(\tilde{p}_{i}, \omega_{i}, \tilde{K}\right)=\tilde{G}$. In the following, I will show that the property $\bar{G}>\tilde{G}$ implies $\bar{g}>\tilde{g}$. For a proof of this claim, assume otherwise, i.e., $\bar{g} \leq \tilde{g}$. This implies $\bar{b} \leq \tilde{b}$. Moreover, $\bar{g}_{j} \leq \tilde{g}_{j}$ and $\bar{x}_{j} \geq \tilde{x}_{j}$ must hold for at least some type $j \in\{1, \ldots, I\}$. Because $\hat{m}_{j}\left(x_{j}, g_{j}, b, K\right)$ is weakly increasing in $K$, and because it is also increasing in $x_{j}$, decreasing in $b$, and weakly decreasing in $g_{j}$ (see the proof of Proposition 2), we thus obtain $\hat{m}_{j}\left(\bar{x}_{j}, \bar{g}_{j}, \bar{b}, \bar{K}\right) \geq \hat{m}_{j}\left(\tilde{x}_{j}, \tilde{g}_{j}, \tilde{b}, \tilde{K}\right)$. Due to the agents' first-order conditions $\hat{m}_{j}(\cdot)=m_{j}(\cdot)=p_{j}(\cdot)$ in the Lindahl equilibrium, this means $\bar{p}_{j} \geq \tilde{p}_{j}$. Because $\bar{g}_{j}=\bar{p}_{j} \bar{G}, \tilde{g}_{j}=\tilde{p}_{j} \tilde{G}$, and $\bar{g}_{j} \leq \tilde{g}_{j}$, this implies $\bar{G} \leq \tilde{G}$ - a contradiction. Therefore, $g^{*}(N, N)$ is increasing in $N$.

\section{Proof of Proposition 4}

Consider first the claim $\lim _{N \rightarrow \infty} e(N, N)=1$. As argued in the main text, some upper bound $\bar{g} \leq \bar{\omega}$ exists with the properties $g^{*}(N, N) \leq \bar{g}$ for all $N \geq 1$ and $\lim _{N \rightarrow \infty} g^{*}(N, N)=\bar{g}$. Assume now that $\lim _{N \rightarrow \infty} e(N, N)=1$ does not hold. Because $g^{v}(N, N)$ is increasing in $N$ (see Proposition 2), we can then find some real number $\gamma<1$ such that $g^{v}(N, N)<\gamma \bar{g}$ for all $N \geq 1$. Since $g^{*}(N, N)$ converges to $\bar{g}$ as $N$ becomes large, there must exist some group size $\hat{N}=\hat{K}$ where $\hat{g}:=g^{*}(\hat{N}, \hat{N}) \geq \gamma \bar{g}$. Let $\left(\hat{x}_{i}, \hat{g}_{i}, \hat{b}, \hat{G}\right)$ denote the corresponding Lindahl allocation. Because of the Samuelson condition (8), we have $\hat{m}_{i}\left(\hat{x}_{i}, \hat{g}_{i}, \hat{b}, \hat{K}\right) \leq 1$ 
for all types $i$. Using the assumption $\lim _{K \rightarrow \infty} \hat{m}_{i}\left(\hat{x}_{i}, \hat{g}_{i}, \hat{b}, K\right)>1$, we can thus find some number $\tilde{K}>\hat{K}$, where $\hat{m}_{i}\left(\hat{x}_{i}, \hat{g}_{i}, \hat{b}, \tilde{K}\right) \geq 1$ holds for all $i \in\{1, \ldots, I\}$. Consider now an economy with size $\tilde{K}=\tilde{N}$. Then it follows immediately from the first-order conditions (4), assumption (6), and the property $\hat{m}_{i}\left(\hat{x}_{i}, \hat{g}_{i}, \hat{b}, \tilde{K}\right) \geq 1$, $i=1, \ldots, I$ that the average transfer $\tilde{b}:=G^{v}(\tilde{N}, \tilde{N}) / \tilde{K}$ in the Nash equilibrium cannot fall below $\hat{b}$. Accordingly, we also have $g^{v}(\tilde{N}, \tilde{N}) \geq \hat{g} \geq \gamma \bar{g}$, which in turn contradicts the claim that $g^{v}(N, N)<\gamma \bar{g}$ holds for all $N \geq 1$. Therefore, $\lim _{N \rightarrow \infty} e(N, N)=1$. Next, note that the inequality $e(N, N)<1$ must hold as long as $N I \geq 2$ (see fn. 18). Hence, $e(2,2)<1=\lim _{N \rightarrow \infty} e(N, N)$, which implies that $e(N, N)$ is increasing in $N$ at least for some $N \geq 1$.

\section{Acknowledgements}

I would like to thank Christoph Engel, Andreas Nicklisch, Ingolf Schwarz, and two referees for very helpful comments on an earlier version of this paper. The usual disclaimer applies.

\section{References}

Abrams, B.A., Schmitz, M.D., 1984. The crowding-out effect of governmental transfers on private charitable contributions: cross-section evidence. National Tax Journal 37, 563-568.

Anderson, S.P., Goeree, J.K., Holt, C.A., 1998. A theoretical analysis of altruism and decision error in public good games. Journal of Public Economics 70, 297-323.

Andreoni, J., 1988. Privately provided public goods in a large economy: the limits of altruism. Journal of Public Economics 35, 57-73.

Andreoni, J., 1990. Impure altruism and donations to public goods: a theory of warm-glow giving. Economic Journal 100, 464-477.

Andreoni, J., Miller, J., 2002. Giving according to GARP: an experimental test of the consistency of preferences for altruism. Econometrica 70, 737-753. 
Becker, G.S., 1974. A theory of social interactions. Journal of Political Economy 82, 1063-1093.

Bergstrom, T., Cornes, R., 1983. Independence of allocative efficiency from distribution in the theory of public goods. Econometrica 51, 1753-1765.

Brunner, E.J., 1998. Free riders or easy riders?: an examination of the voluntary provision of public radio. Public Choice 97, 587-604.

Buchholz, W., Peters, W., 2001. The overprovision anomaly of private public good supply. Journal of Economics 74, 63-78.

Chamberlin, J., 1974. Provision of collective goods as a function of group size. American Political Science Review 68, 707-716.

Cornes, R., Hartley, R., Sandler, T., 1999. Equilibrium existence and uniqueness in public good models: an elementary proof via contraction. Journal of Public Economic Theory 1, 499-509.

Cornes, R., Sandler, T., 1984. Easy riders, joint production, and public goods. Economic Journal 94, 580-598.

Cornes, R., Sandler, T., 1996. The Theory of Externalities, Public Goods, and Club Goods, 2nd ed. Cambridge University Press, Cambridge.

Fries, T.L., Golding, E., Romano, R., 1991. Private provision of public goods and the failure of the neutrality property in large finite economies. International Economic Review 32, 147-157.

Gaube, T., 2001. Group size and free riding when private and public goods are gross substitutes. Economics Letters 70, 127-132.

Goeree, J.K., Holt, C.A., Laury, S.K., 2002. Private costs and public benefits: unravelling the effects of altruism and noisy behavior. Journal of Public Economics 83, 255-276.

Haan, M., Kooreman, P., 2002. Free riding and the provision of candy bars. Journal of Public Economics 83, 277-291.

Isaac, R.M., Walker, J.M., 1988. Group size effects in public goods provision: the voluntary contribution mechanism. Quarterly Journal of Economics 103, 179-200. 
Isaac, R.M., Walker, J.M., Williams, A.W., 1994. Group size and the voluntary provision of public goods: experimental evidence utilizing large groups. Journal of Public Economics 54, 1-36.

McGuire, M.C., 1974. Group size, group homogeneity, and the aggregate provision of a pure public good under Cournot behavior. Public Choice 18, 107-126.

Lipford, J., 1995. Group size and the free-rider hypothesis: an examination of new evidence from churches. Public Choice 83, 291-303.

Olson, M., 1965. The Logic of Collective Action. Harvard University Press, Cambridge/MA.

Ribar, D.C., Wilhelm, M.O., 2002. Altruism and joy-of-giving motivations in charitable behavior. Journal of Political Economy 110, 425-457.

Sandler, T., 1992. Collective Action. Theory and Applications. Harvester Wheatsheaf, New York. 